\title{
The COVID-19 pandemic should be last orders for poor care of people with neurodevelopmental disorders
}

\author{
Andre Strydom, Elizabeth Corcoran and Anne-Sophie Rebillat
}

\section{Summary}

We explore whether the needs of individuals with neurodevelopment disorders have been overlooked during the coronavirus disease 2019 (COVID-19) pandemic and set out the issues that need to be considered in response to future health crises and pandemics.

\section{Keywords}

COVID; neurodevelopmental disorders; carers; intellectual disability; autism spectrum disorders.

\section{Copyright and usage}

(c) The Author(s), 2021. Published by Cambridge University Press on behalf of the Royal College of Psychiatrists. This is an Open Access article, distributed under the terms of the Creative Commons Attribution licence (http://creativecommons.org/ licenses/by/4.0/), which permits unrestricted re-use, distribution, and reproduction in any medium, provided the original work is properly cited.
Andre Strydom (pictured) is a psychiatrist and clinical academic in intellectual disabilities at the Institute of Psychiatry, Psychology, and Neuroscience, King's College London and the South London and Maudsley Foundation NHS Trust. He is the president of the Trisomy 21 Research Society. Elizabeth Corcoran is a psychiatrist and Chair of the Down's Syndrome Research Foundation and is passionate about advocacy and empowering the community to support research. Anne-Sophie Rebillat is a geriatrician. Within the Jérôme Lejeune Institute in Paris, she runs a clinic specialised in the management of age-related diseases for patients with Down syndrome. Her research interest is mainly focused on comorbidities of cognitive functioning with ageing ( such as Alzheimer's disease and obstructive sleep apnoea) in people with Down syndrome.

\section{Background}

The chaotic initial stages of the SARS-CoV-2 pandemic, which threatened to overwhelm health services in many regions in the northern hemisphere in the spring of 2020, exposed an uncomfortable truth: the most vulnerable individuals, including those with neurodevelopmental disorders (NDD), remain at the margins of society and their risks, health and mental health needs during emerging pandemics are consequently overlooked. Many clinicians, patients and caregivers were surprised that a so-called herd immunity approach could be considered by politicians (and some scientists) without giving consideration to which members of our communities might be more severely affected before this is achieved.

When the reality of the first wave of coronavirus disease 2019 (COVID-19) became fully apparent, a lack of planning for protecting those at most risk, insufficient resources for care settings outside of hospitals and hasty legislation seemed to further disadvantage individuals with NDDs. Governments and policymakers are not solely to blame, however - some scientists and particularly data modellers demonstrated a remarkable blind spot to the likely additional risk associated with disabilities such as NDD. Most of the highly publicised modelling studies did not fully take into account the risk for those with disabilities, or the circumstances in which many of these individuals are being cared for, with the result that these studies have almost without exception significantly underestimated the impact of the pandemic in these communities.

\section{Gaps in understanding of risks, presentation of disease and outcomes}

During the initial stages NDD communities grappled with the question 'am I/they vulnerable?' 'should I shield?' based solely on their diagnosis of NDD. Given the gaps in basic immunology and physiological research into their conditions, combined with the absence of widespread testing, clinicians and community leaders could not provide them with evidence-based advice about extra protection or shielding. Survey data has, however, provided a valuable snapshot of NDD communities highlighting that rates of multimorbidity and poly-pharmacy are still predominant, suggesting an increased risk for more severe COVID-19 (https://www.dsrf-uk.org/covid19results2/).

During the course of the pandemic the high risk to individuals with NDDs have become increasingly apparent. For example, people with Down syndrome were shown to have three times the mortality risk after developing COVID-19 symptoms, and at a much younger age, than their peers in the general population. These were associated with their increased rates of comorbidities such as Alzheimer's disease and obesity as well as innate immune system dysfunction. ${ }^{1}$ Other individuals with NDD particularly those with intellectual disabilities, also appear to be at increased risk for both admission to hospital and mortality because of COVID-19. ${ }^{2}$ It is not yet clear to what extent this increased risk is explained by environmental factors such as living in care settings and difficulty following safety measures such as hand-washing or using face masks, constitutional factors such as comorbidities and immune system issues, or because of individuals with NDD being disadvantaged at service-delivery level, for example, not having equal access to interventions such as ventilation.

A particular concern in the UK was the proposal to use the clinical frailty scale as a decision tool to prioritise care, without consideration of how it might be applied to individuals with NDD. With a focus on functional abilities and lack of adjustment for quality of life, application of this scale in its original form would have meant that individuals with stable cognitive impairments may not have been deemed a priority for intervention, causing distress in the intellectual disability community and fuelling fears of utilitarian priorities in healthcare. Fortunately, a robust response from the NDD community resulted in the deeply flawed approach being adapted. 


\section{Care homes}

The fragmentation of the care sector (and Government agencies that have responsibility for overseeing it) in many countries including the UK and the USA further complicated efforts to coordinate the response to protect people with NDD. Responsibility to provide personal protective equipment (PPE) and to keep track of cases in settings such as smaller care homes typically provided by the private sector was not clear. In some cases, this had disastrous consequences, most infamously in Spain where there were reports of people in care homes being left abandoned (https://www.bbc.co. $\mathrm{uk} /$ news/world-europe-52014023). In contrast to the piecemeal approach to public health in the UK following the transfer of public health services from the National Health Service to local authorities in 2012, Germany has maintained a public health institute, which arguably helped it to achieve a more coherent response. ${ }^{3}$

\section{Impact on quality of life}

There have also been examples of exceptional care, with caregivers going above and beyond what is expected to support people with NDD; for example, moving in with the vulnerable person during the lockdown so as to limit exposure through employing multiple caregivers. Some large care organisations in the USA mobilised early, and developed systems to monitor outbreaks in individual homes and implemented a rapid response. ${ }^{4}$ Others eventually implemented regular testing for care workers to limit exposure to residents, and most care homes followed strict social distancing guidelines. This successfully limited the infection rate in many facilities. However, it came at a cost - many people with NDD, who benefit from the structure and predictability of a regular routine, found the change in their daily routines challenging, and others had to forego face-to-face contact with families and friends. Given that people with NDD and their families often have limited social networks, the requirement for social distancing may have resulted in significant social isolation. Furthermore, organised social, educational and occupational activities are often an essential part of care plans to maintain mental and physical health and keep people active. The loss of these activities during the lockdown may have resulted in increased functional impairment, as well as an increase in mental health and behavioural issues. Whether this will affect longer-term mental and physical health outcomes remains unknown.

\section{Planning for the future}

For all of these reasons, it is of utmost importance to include a comprehensive consideration of the needs of individuals with NDD in planning our response to future health crises and pandemics such as COVID-19. We suggest an urgent focus on improving and coordinating efforts to prevent, detect, contain and treat infections involving people with NDD during the current and future pandemics, while addressing mental health and quality of life concerns.

First, to prevent more severe outcomes, early mobilisation and a coordinated response is essential to safeguard vulnerable individuals with NDD during pandemics. To achieve this, structures for live data-sets from populations with NDD should be set up that can be rapidly employed to understand their risk, differences in presentation and outcomes, including the risk for mental health and behavioural issues as a consequence of containment or isolation. The longerterm consequences of pandemics, for example, of the so-called 'long' COVID should also be monitored in NDD communities. Such data should be incorporated into efforts to model disease outcomes and the potential impact of interventions at population level.
Pandemic models are unlikely to be valid unless the risk associated with disabilities including NDD are specifically considered.

Second, people with NDD, particularly those living in conglomerate settings, should be prioritised for detection of infection, which will be aided by development of non-invasive and rapid testing. This must include regular testing of care staff and caregivers. There should be coordination of data on outbreaks within care settings to understand outbreaks at both local and national levels. Transmission in long-term care facilities should be contained with a rapid response to outbreaks, including containment and prioritisation for the provision of adequate PPE. Once people with NDD have contracted an infection, caregivers should be supported to provide close monitoring and safe care, and to identify symptoms that may indicate the need for admission to hospital and active treatment. Appropriate medical management with easy access to oxygen therapy and drugs such as steroids, antibiotics and anticoagulants at home could avoid complications and hospital admissions.

Within hospital settings, healthcare staff need to urgently address discriminating practices and ensure appropriate and equal access to treatment in keeping with the individual's clinical status. Diagnostic overshadowing remains a phenomenon we must all remain vigilant against in the care of those with NDDs. There are many of our colleagues that have little to no training in providing care or previous exposure to those with NDDs. Families and carers must be reassured that they are generally permitted to remain with their loved one when admitted to facilitate access to healthcare. Ideally all hospitals would have intellectual disability specialists/nurses to guide the delivery of healthcare to those with NDD and support the professionals to deliver high-quality care.

Third, people with NDDs, particularly those with additional risk factors, should be prioritised for treatment and interventions, including immunisation with the coronavirus vaccine. In order to facilitate this, people with NDD should be included in vaccine trials, as there may be a different antibody response in some groups. In addition to the flu vaccine, individuals such as those with Down syndrome who have an increased rate of death because of pneumonia may also benefit from pneumococcal vaccination. Many people with NDDs are deficient in Vitamin D, particularly during winter months, and supplementation may be beneficial during pandemics involving respiratory pathogens given some evidence that it may modulate host responses to respiratory infections. ${ }^{5}$

Last but not least, it is important to consider the mental health impact of pandemics, including the potential impact of lockdowns and shielding. Loss of structured activities should be replaced with alternatives during lockdowns, and individuals with NDD should be monitored for signs of mental health concerns. There are concerns that the fall-out from the current pandemic may include loss of funding for facilities and supports on which individuals with NDD and their caregivers depend to maintain their mental health and quality of life; clinicians working with individuals with NDD will need to strongly advocate for their continued access to such support. From a human rights perspective, public health policies should benefit vulnerable people too, and not solely focus on maintaining the economy. However, the decisions taken today in terms of public health should not only be based on the conditions of the first wave, but also take into account the progress made in medical care with reduction in mortality rates, and the capacity of people with NDD to respect health rules.

\section{Conclusions}

There have been many lessons learned during the initial stages of the COVID-19 pandemic - most importantly, that without a focus on 
vulnerable people we cannot fully estimate the impact of disease at population level.

Andre Strydom (1D, King's College London, UK; and South London and the Maudsley Foundation NHS Trust, UK; Elizabeth Corcoran, Down's Syndrome Research

Foundation, UK; Anne-Sophie Rebillat, Jérôme Lejeune Institute, France

Correspondence: Andre Strydom. Email: andre.strydom@kcl.ac.uk

First received 9 Nov 2020, final revision 14 Dec 2020, accepted 01 Feb 2021

\section{Author contribution}

A.S. E.C. and A.S.R. co-wrote this editorial.

\section{Declaration of interest}

A.S. is president of the Trisomy 21 Research Society; E.C. runs the Down's Syndrome Research Foundation.

\section{References}

1 Huels A, Costa ACS, Dierssen M, Baksh RA, Bargagna S, Baumer NT, et al. An international survey on the impact of COVID-19 in individuals with Down syndrome. medRxiv [Preprint] 2020. Available from: https://doi.org/10.1101/2020. 11.03.20225359.

2 NHS England. COVID-19 Deaths of Patients with a Learning Disability Notified to LeDeR. NHS England, 2020 (https://www.england.nhs.uk/publication/covid19-deaths-of-patients-with-a-learning-disability-notified-to-leder/).

3 Our World in Data. Emerging COVID-19 Success Story: Germany's Strong Enabling Environment. Our World in Data, 2020 (https://ourworldindata.org/ covid-exemplar-germany)

4 Mills WR, Sender S, Lichtefeld J, Romano N, Reynolds K, Price M, et al. Supporting individuals with intellectual and developmental disability during the first 100 days of the COVID-19 outbreak in the USA. J IntellI Disab Res 2020; 64: 489-96.

5 Martineau AR, Forouhi NG. Vitamin D for COVID-19: a case to answer? Lancet Diabetes Endocrinol 2020; 8: 735-6. 